\title{
The Gas Diffusion Electrode Setup as Straightforward Testing Device for Proton Exchange Membrane Water Electrolyzer Catalysts
}

\author{
Johanna Schröder, Vladislav A. Mints, Aline Bornet, Etienne Berner, Mohammad Fathi Tovini, \\ Jonathan Quinson, Gustav K. H. Wiberg, Francesco Bizzotto, Hany A. El-Sayed, and Matthias Arenz*
}

Cite This: JACS Au 2021, 1, 247-251

Read Online

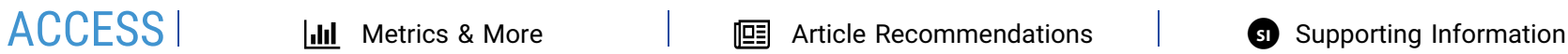

ABSTRACT: Hydrogen production from renewable resources and its reconversion into electricity are two important pillars toward a more sustainable energy use. The efficiency and viability of these technologies heavily rely on active and stable electrocatalysts. Basic research to develop superior electrocatalysts is commonly performed in conventional electrochemical setups such as a rotating disk electrode (RDE) configuration or H-type electrochemical cells. These experiments are easy to set up; however, there is a large gap to real electrochemical conversion devices such as fuel cells or electrolyzers. To close this gap, gas diffusion electrode (GDE) setups were recently presented as a straightforward technique for testing fuel cell catalysts under more realistic conditions. Here, we demonstrate for the first time a GDE setup for measuring the oxygen evolution reaction (OER) of catalysts for proton exchange membrane water electrolyzers (PEMWEs). Using a commercially available benchmark $\mathrm{IrO}_{2}$ catalyst deposited on a carbon gas diffusion layer (GDL), it is shown that key

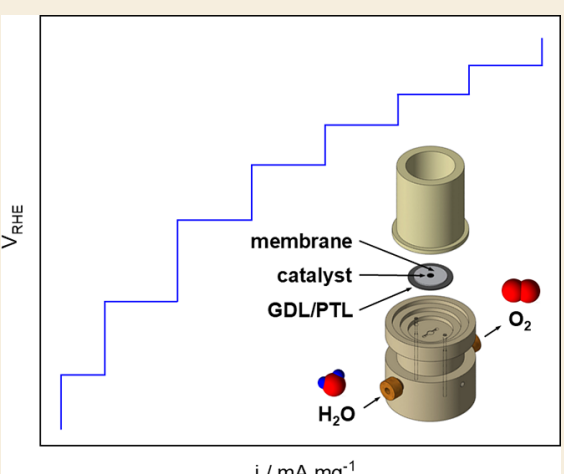
parameters such as the OER mass activity, the activation energy, and even reasonable estimates of the exchange current density can be extracted in a realistic range of catalyst loadings for PEMWEs. It is furthermore shown that the carbon-based GDL is not only suitable for activity determination but also short-term stability testing. Alternatively, the GDL can be replaced by Ti-based porous transport layers (PTLs) typically used in commercial PEMWEs. Here a simple preparation is shown involving the hot-pressing of a Nafion membrane onto a drop-cast glycerol-based ink on a Ti-PTL.

KEYWORDS: oxygen evolution reaction, gas diffusion electrode setup, water electrolyzer, iridium-based catalysts, Ti porous transport layer, performance screening

EMWEs coupled to renewable energy sources such as wind and solar are a promising technology for energy conversion and long-term storage because of their higher current density as compared with alkaline electrolysis cells (AECs) ${ }^{1-3}$ For PEMWEs, the preferred anode materials are Ir and Ir alloys because of the combination of high activity and stability of $\mathrm{IrO}_{2} \cdot{ }^{4-6}$ As $\mathrm{Ir}$ is one of the rarest precious metals, an efficient testing platform requiring only small amounts of Ir to screen PEMWE catalysts is necessary to optimize the anode performance. In this work, an in-house developed GDE setup $^{8-11}$ previously used for oxygen reduction reaction (ORR) studies is adapted to enable OER measurements. In this modified setup, the stainless-steel body is replaced by polychlorotrifluoroethylene for enhanced chemical stability at high voltages (see Figure 1 for the scheme of the developed GDE setup for straightforward OER measurements and Figures S1-S2).

The GDE consists of a catalyst layer deposited on top of a carbon GDL by vacuum filtration as introduced by Yarlagadda et al. ${ }^{12}$ The vacuum filtration enables a reproducible film quality with catalyst loadings comparable to PEMWEs. ${ }^{7,13}$ Element mapping by energy-dispersive X-ray spectroscopy

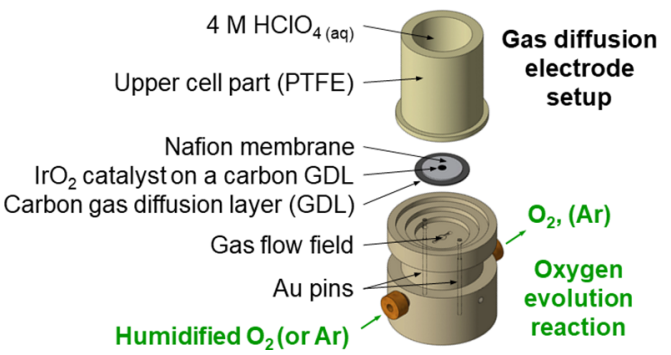

Figure 1. Scheme of the developed GDE setup for straightforward OER measurements.

(EDS) reveals a clearly separated $\mathrm{IrO}_{2}$ layer on top of the microporous carbon layer (MPL) of the GDL (see Figure 2).

Published: February 17, 2021

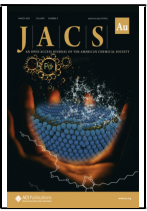



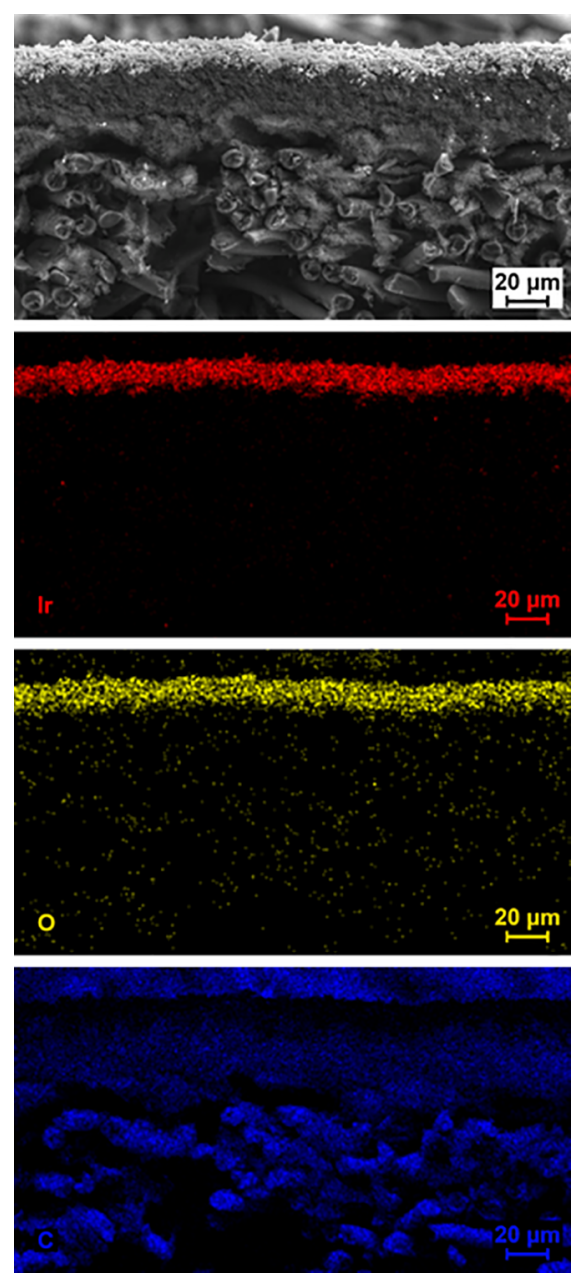

Figure 2. SEM cross-section of an activated $\mathrm{IrO}_{2}$ catalyst film deposited on a GDL in combination with EDS mapping of iridium (Ir, red), oxygen ( $\mathrm{O}$, yellow), and carbon ( $\mathrm{C}$, blue). A clear separation of the catalyst film, the carbon containing MPL, and the carbon fibers of the GDL is apparent.

To establish a measurement protocol for the new GDE setup, the influence of several experimental parameters such as the gas flow rate, gas atmosphere $\left(\mathrm{Ar}, \mathrm{O}_{2}\right)$, substrate, and temperature on the measured reaction rate were investigated (see Table $\mathrm{S} 1$ for a summary). In addition, the results were compared to conventional RDE measurements. ${ }^{14-17}$

The influence of the reactant gas flow (humidified Ar) on the observed OER activity is shown in Figure S4. Based on the measurements, possible mass transport limitations can be identified. The results demonstrate that the apparent OER activities and Tafel slopes are relatively constant $(41.4 \pm 0.9$ $\mathrm{mV} \mathrm{dec}{ }^{-1}$ ) over a wide range of the reactant gas flow (40-190 $\left.\mathrm{mL} \mathrm{min}^{-1}\right)$. Only for low flow rates $\left(10 \mathrm{~mL} \mathrm{~min}^{-1}\right)$, the observed OER rates are inhibited and the Tafel slope shows nonlinear behavior. These observations indicate mass transport limitations at too low flow rates because of a lack of water transported to the catalyst or the difficulty in removing produced $\mathrm{O}_{2}$ from the surface. Hence, in the following, a flow rate of $40 \mathrm{~mL} \mathrm{m^{-1 }}$ was used to ensure sufficient reactant mass transport.

On the basis of the investigations, we established a measurement protocol, where a set current (normalized to the mass of the precious metal catalyst) is applied and the electrode potential is recorded as a function of time (see Figure S4). Given the controlled current, the average of the $i R$ corrected potential of the last $60 \mathrm{~s}$ (when not stated differently) of every step is defined as the OER.

Thus, conducted OER measurements with a commercial $\mathrm{IrO}_{2}$ benchmark catalyst are presented in Figure 3. In Figure

a

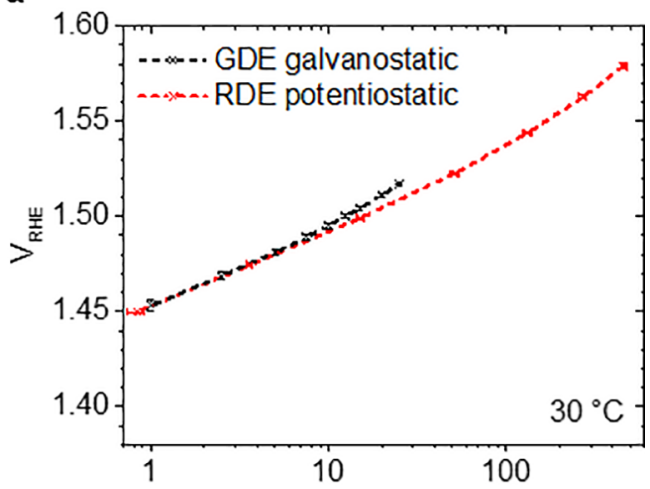

b

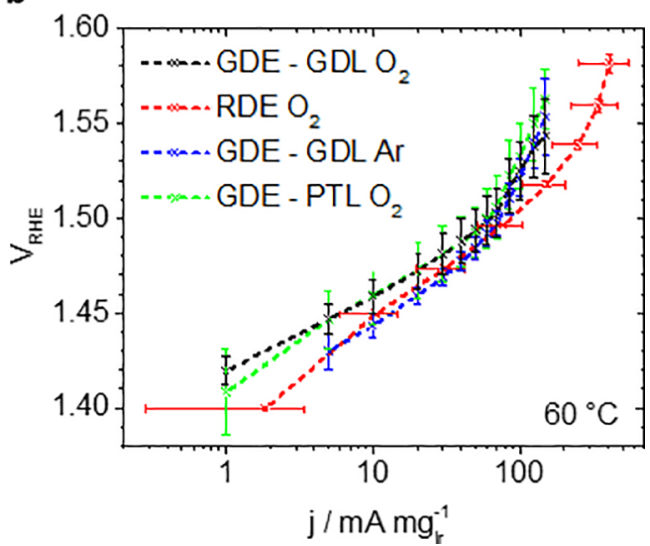

Figure 3. Tafel plots of OER mass activity $j$ of the $\mathrm{IrO}_{2}$ catalyst. Comparison of GDE (galvanostatic, $4 \mathrm{M} \mathrm{HClO}_{4}, 1 \mathrm{mg}_{\mathrm{Ir}} \mathrm{cm}^{-2}$ geo ) and $\mathrm{RDE}$ (potentiostatic in $\mathrm{O}_{2}$ atmosphere, $0.1 \mathrm{M} \mathrm{HClO}_{4}, 50 \mu \mathrm{g}_{\text {Ir }}$ $\mathrm{cm}^{-2}$ geo $)$ measurements (a) at $30{ }^{\circ} \mathrm{C}$ in $\mathrm{O}_{2}$ and (b) $60{ }^{\circ} \mathrm{C}$ using a GDL in $\mathrm{O}_{2}$ and $\mathrm{Ar}$ or Ti-PTL in $\mathrm{O}_{2}$ atmosphere in the GDE setup. The error bars show the standard deviation of three independent measurements; the data points are connected by dashed lines as guide for the eye.

3a, we compare GDE measurements to conventional RDE measurements at $30{ }^{\circ} \mathrm{C}$ in an $\mathrm{O}_{2}$ atmosphere. It is seen that the determined mass activities and Tafel slopes are similar in both setups. At $1.48 \mathrm{~V}_{\mathrm{RHE}}$, mass activities of 4.3 and $4.9 \mathrm{~mA} \mathrm{mg}_{\mathrm{Ir}}{ }^{-1}$ are determined for the galvanostatic GDE and potentiostatic RDE measurements, respectively, which is comparable to the values reported by Alia et al. ${ }^{18,19}$ The determined Tafel slopes are $45.4 \pm 1.0$ and $39.2 \pm 0.4 \mathrm{mV} \mathrm{dec}^{-1}$, respectively.

In Figure $3 \mathrm{~b}$, the results at an increased temperature of 60 ${ }^{\circ} \mathrm{C}$ are shown. In a $\mathrm{RDE}$ setup, potentiodynamic measurements reveal performance degradation during continuous cycling (see Figure S5), and potentiostatic measurements show a large standard deviation for the measured current density. By comparison, in the GDE setup, three independent measurements confirm a good reproducibility of the obtained OER activities under steady-state conditions (see also Table $\mathrm{S} 1$ ). The fast performance degradation, as seen in the RDE, is most likely inhibited in the GDE setup because of the use of a 
Nafion membrane avoiding direct contact of the catalyst to the liquid electrolyte leading to a more realistic setup in the GDE comparable to a PEMWE. Furthermore, it is demonstrated that the OER activity can be conveniently studied in $\mathrm{O}_{2}$ or Ar gas atmosphere. The latter leads to apparent activity improvements, which can be understood by the influence of the $\mathrm{O}_{2}$ partial pressure in the Nernst equation. The same effect influences the Tafel behavior. In $\mathrm{O}_{2}$ atmosphere and $60{ }^{\circ} \mathrm{C}$, a Tafel slope of $41.1 \pm 4.8 \mathrm{mV} \mathrm{dec}^{-1}$ is observed at low current densities, which at higher current densities increases significantly. This observation is linked to oxygen bubble formation, which leads to inhibited OER rates. ${ }^{20,21}$ As a consequence, with increasing oxygen formation rates (i.e., higher current densities), the apparent OER activity in Ar atmosphere decreases and approaches that in $\mathrm{O}_{2}$. Interestingly, nonlinearities in the Tafel slope at low current densities, which are often observed in potentiodynamic RDE measurements, ${ }^{22}$ are not apparent in the GDE setup.

Most importantly, no fundamental difference is observed if these OER activity measurements are performed with a catalyst film deposited onto a carbon GDL or Ti-PTL, justifying the substantially less complex GDL approach for catalyst screening; see more details about the film preparation in the SI. To further corroborate that carbon corrosion of the GDL does not pose a problem during the activity measurement, the current density was increased stepwise from 1 to $10 \mathrm{~mA} \mathrm{mg}{ }_{\text {Ir }}^{-1}$ and held at $10 \mathrm{~mA} \mathrm{mg}_{\mathrm{Ir}}{ }^{-1}$ for $1 \mathrm{~h}$. Figure 4 shows that even with the

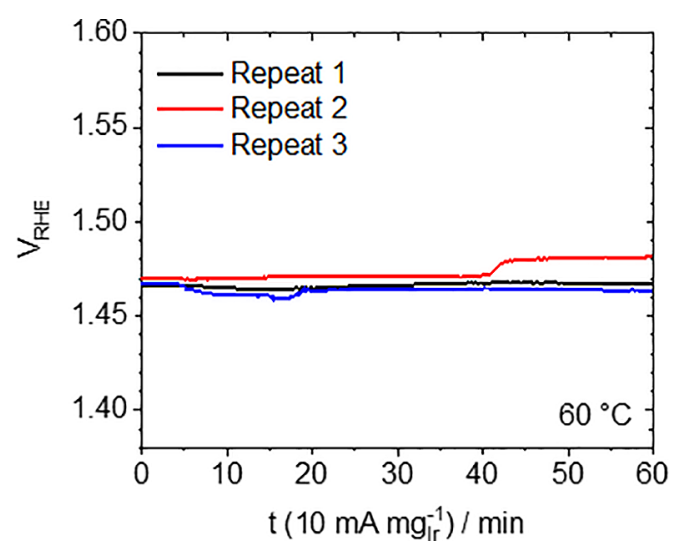

Figure 4. Stability measurements of $\mathrm{IrO}_{2} / \mathrm{GDL}$ applying $10 \mathrm{~mA}$ $\mathrm{mg}_{\text {Ir }}^{-1}$ for $1 \mathrm{~h}$.

carbon-based GDL, it is possible to perform such suggested stability measurements, ${ }^{23}$ although the potential increase in repeat 2 (of three independent samples) can probably be traced back to bubble formation or carbon degradation. Hence the GDL is a suitable substrate for determining the activity of OER catalysts in screening-type studies.

For long-term stability measurements under harsher conditions, catalyst-coated membranes (CCMs) pressed to Ti-PTLs might be used. ${ }^{24,25}$ However, CCM preparation is time-consuming and needs special equipment; hence, it is not available in all laboratories for a fast screening test protocol. Because of the high porosity of the PTL, vacuum filtration or drop-casting using an ink of water and IPA as for the GDLs preparation is not possible. A simple alternative to CCM preparation is to use a glycerol-based catalyst ink. To completely avoid the ink permeation into the PTL, Teflon was sprayed on top of the PTL before drop-casting (note that the MPL on top of the carbon fiber structure of the GDL also contains Teflon). Furthermore, as compared with the GDL preparation, the temperature during pressing needs to be increased; otherwise, a poor electric connection between Nafion membrane and PTL can lead to high resistances of several hundred ohms. However, a homemade hot-press was sufficient to enable the use of a PTL and achieve results comparable to the GDL approach (see Figure 5b). Therefore,
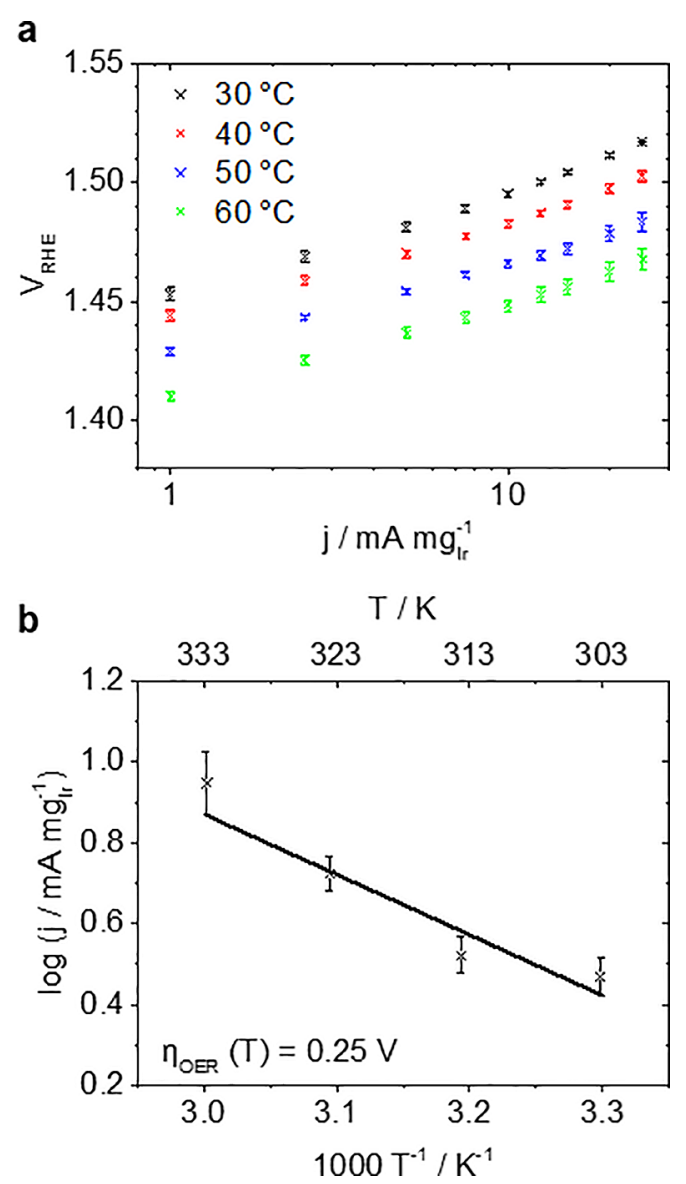

Figure 5. (a) Temperature-dependent OER mass activity $j$ plotted as Tafel plots obtained in a GDE setup applying current steps versus $V_{\mathrm{RHE}}$ (b) The corresponding Arrhenius plot at an overpotential $\eta_{\mathrm{OER}}$ of $0.25 \mathrm{~V}$.

the use of a PTL is possible in the presented GDE setup even without specialized equipment. However, because of the simple hot-pressing, the membrane can peel off with time at high $\mathrm{O}_{2}$ formation rates. Additionally, because of the high costs of the PTL, we believe that the GDL approach is more suitable for a first activity screening.

To complete the $\mathrm{IrO}_{2}$ catalyst characterization, the temperature dependency was investigated between 30 and $60{ }^{\circ} \mathrm{C}$. In Figure 5a, it is shown that, as expected, the OER rate increases with temperature. ${ }^{26}$ Furthermore, comparable Tafel slopes (38.5-45.4 $\left.\mathrm{mV} \mathrm{dec}^{-1}\right)$ are observed, indicating that the OER reaction mechanism is the same in the investigated temperature range. Hence, the electrochemical activation energy $E_{\mathrm{A}}$ can be estimated assuming Arrhenius behavior. For a rough estimation, the formal $E_{\mathrm{A}}$ determined with respect to the reference potential of the used reversible hydrogen reference electrode (RHE) can be used (see SI). For a more precise determination, the temperature-dependent shift of the 
reversible oxygen potential versus RHE needs to be considered. This correction is also required when determining the exchange current density $j_{0}$. The precise Tafel slope allows for an extrapolation of the linear fits to the reversible potential to determine $j_{0}$ for the OER. In conventional, potentiodynamic RDE measurements, determining $j_{0}$ for the OER is daring and usually not pursued because an extrapolation of the measured current densities over several orders of magnitude is required. Error margins in the Tafel slope therefore can lead to substantial uncertainties of several orders of magnitude. For the determination of the temperature-dependent reversible potential $E_{\text {rev,T }}$ different equations can be found (e.g., from Parthasarathy et al. ${ }^{27}$ or from Bratsch). ${ }^{28}$ The latter contains an approximation of the exact dependence. Determining $E_{\text {rev, } T}$ using the equation of Parthasarathy et al. ${ }^{27} j_{0}$ of $10.6 \pm 6.0,3.5$ $\pm 1.8,2.1 \pm 1.2$, and $7.2 \pm 3.8 \times 10^{-9} \mathrm{~A} \mathrm{mg}_{\mathrm{Ir}}^{-1}$ are obtained for $30,40,50$, and $60^{\circ} \mathrm{C}$, respectively. It should be noted that three independent samples were measured while consecutively applying the four temperatures. Determining $E_{\text {rev,T }}$ following Bratsch $^{28}$ leads to comparable but slightly higher values of $j_{0}$ (see Tables S2-S3). The obtained $j_{0}$ are comparable to $7.3 \times$ $10^{-9} \mathrm{~A} \mathrm{mg}_{\mathrm{Ir}}{ }^{-2}$ calculated from the data reported by $\mathrm{Lu}$ et al. ${ }^{22}$ The limitation of the extrapolation, however, is apparent from the increase in $j_{0}$ going from 50 to $60{ }^{\circ} \mathrm{C}$. For the determination of $E_{\mathrm{A}}$ at constant overpotential, $E_{\mathrm{rev}, \mathrm{T}}$ has been calculated according to Parthasarathy et al. ${ }^{27}$ (see Figure S6 for a Arrhenius plot according to the correction by Bratsch $^{28}$ ). The linear fit of the Arrhenius plot at an overpotential $\eta_{\text {OER }}$ of $0.25 \mathrm{~V}$ (Figure $5 \mathrm{~b}$ ) leads to $E_{\mathrm{A}}$ of $28.5 \pm 6.6 \mathrm{~kJ} \mathrm{~mol}^{-1}$ being a bit lower than $47 \mathrm{~kJ} \mathrm{~mol}^{-1}$ reported by Suermann et al. ${ }^{29}$ in a PEMWE, which is mainly related to the point at $30{ }^{\circ} \mathrm{C}$. Using kinetic data of four temperatures certainly only allows a rough estimation. Nevertheless, this example shows the potential of the presented GDE approach to provide high quality data.

To sum up, we demonstrate a new strategy to test OER catalysts in a GDE setup. The GDE setup is a more straightforward technique than $\mathrm{RDE}^{10,11}$ and the GDE cell can be built in any research workshop (see technical drawings in SI). Depositing the catalyst onto a carbon GDL is suitable for OER steady-state activity screening and allows the determination of key kinetic data. In contrast to conventional potentiodynamic RDE measurements, the catalyst layers contain Nafion and exhibit realistic loadings comparable to PEMWEs. Accurate Tafel slopes can be obtained and extrapolated to determine the exchange current density. Even short-term stability tests are feasible with carbon GDL, which are cost-efficient substrates for catalyst screening. Furthermore, the GDE setup can be used for more elaborate stability screenings using Ti-PTLs as substrate and performing a simple hot-pressing procedure.

\section{ASSOCIATED CONTENT}

\section{Supporting Information}

The Supporting Information is available free of charge at https://pubs.acs.org/doi/10.1021/jacsau.1c00015.

Experimental (chemicals, materials, and gases; gas diffusion electrode setup; rotating disk electrode setup; ink formation; catalyst film preparation; electrochemical measurements; characterization), OER activity measurements (GDE and RDE data; Tafel plot determination; exchange current density and activation energy), XPS and TEM measurements (PDF)

\section{AUTHOR INFORMATION}

Corresponding Author

Matthias Arenz - Department of Chemistry, Biochemistry and Pharmaceutical Sciences, University of Bern, 3012 Bern, Switzerland; 이이.org/0000-0001-9765-4315; Email: matthias.arenz@dcb.unibe.ch

\section{Authors}

Johanna Schröder - Department of Chemistry, Biochemistry and Pharmaceutical Sciences, University of Bern, 3012 Bern, Switzerland; 이이.org/0000-0001-5461-4751

Vladislav A. Mints - Department of Chemistry, Biochemistry and Pharmaceutical Sciences, University of Bern, 3012 Bern, Switzerland

Aline Bornet - Department of Chemistry, Biochemistry and Pharmaceutical Sciences, University of Bern, 3012 Bern, Switzerland; 이이.orcid.o000-0001-9850-2735

Etienne Berner - Department of Chemistry, Biochemistry and Pharmaceutical Sciences, University of Bern, 3012 Bern, Switzerland

Mohammad Fathi Tovini - Chair of Technical Electrochemistry, Department of Chemistry and Catalysis Research Center, Technical University Munich, 85748 Garching, Germany

Jonathan Quinson - Department of Chemistry, University of Copenhagen, 2100 Copenhagen, Denmark; ㅈorcid.org/ 0000-0002-9374-9330

Gustav K. H. Wiberg - Department of Chemistry, Biochemistry and Pharmaceutical Sciences, University of Bern, 3012 Bern, Switzerland

Francesco Bizzotto - Department of Chemistry, Biochemistry and Pharmaceutical Sciences, University of Bern, 3012 Bern, Switzerland

Hany A. El-Sayed - Chair of Technical Electrochemistry, Department of Chemistry and Catalysis Research Center, Technical University Munich, 85748 Garching, Germany; (1) orcid.org/0000-0002-8769-8258

Complete contact information is available at: https://pubs.acs.org/10.1021/jacsau.1c00015

\section{Author Contributions}

G.K.H.W. designed the GDE setup for the OER measurements. J.S., M.A., H.A.E.-S. designed the electrochemical experiments in the GDE setup, prepared and performed by J.S. V.A.M. performed the RDE measurements. J.S. prepared the samples for TEM and XPS measurements. J.Q. performed TEM and M.F.T. XPS measurements and analysis. E.B. did the SEM and EDS measurements. A.B. did initial tests on the hot pressing. F.B. did initial OER tests in the GDE setup. H.A.E.-S. and M.A. supervised the research. J.S. and M.A. wrote the first draft of the paper, read, and commented by all authors.

Notes

The authors declare no competing financial interest.

\section{ACKNOWLEDGMENTS}

This Project has received funding from the European Union's Horizon 2020 Research and Innovation program under grant agreement N. 861960 ("Recycalyse" project) and the Swiss National Science Foundation (SNSF) via the project No. 200021_184742. J.Q acknowledges the European Union's Horizon 2020 research and innovation program under the 
Marie Skłodowska-Curie grant agreement No. 840523 (CoSolCat). J.Q. and M.A. thank Dr. L. Theil Kuhn and Dr. S. B. Simonsen, Technical University of Denmark (DTU) for access to TEM facilities. H.A.E.-S. and M.F.T. gratefully acknowledge the German Ministry of Education and Research for financial support of this work within the innoKA project (BMWi, 03ET6096A). Dr. Nedjeljko Seselj of Danish Power Systems (DPS) is acknowledged for his advice concerning the catalyst film formation on the PTL.

\section{REFERENCES}

(1) Buttler, A.; Spliethoff, H. Current Status of Water Electrolysis for Energy Storage, Grid Balancing and Sector Coupling via Power-toGas and Power-to-Liquids: A Review. Renewable and Sustainable Energy Reviews 2018, 82, 2440-2454.

(2) Babic, U.; Suermann, M.; Büchi, F. N.; Gubler, L.; Schmidt, T. J. Critical Review-Identifying Critical Gaps for Polymer Electrolyte Water Electrolysis Development. J. Electrochem. Soc. 2017, 164 (4), F387-F399.

(3) Schmidt, O.; Gambhir, A.; Staffell, I.; Hawkes, A.; Nelson, J.; Few, S. Future Cost and Performance of Water Electrolysis: An Expert Elicitation Study. Int. J. Hydrogen Energy 2017, 42 (52), 30470-30492.

(4) Fabbri, E.; Habereder, A.; Waltar, K.; Kötz, R.; Schmidt, T. J. Developments and Perspectives of Oxide-Based Catalysts for the Oxygen Evolution Reaction. Catal. Sci. Technol. 2014, 4 (11), 38003821.

(5) Antolini, E. Structural Parameters of Supported Fuel Cell Catalysts: The Effect of Particle Size, Inter-Particle Distance and Metal Loading on Catalytic Activity and Fuel Cell Performance. Applied Catalysis B: Environmental 2016, 181, 298-313.

(6) McCrory, C. C. L.; Jung, S.; Peters, J. C.; Jaramillo, T. F. Benchmarking Heterogeneous Electrocatalysts for the Oxygen Evolution Reaction. J. Am. Chem. Soc. 2013, 135 (45), 16977-16987.

(7) Carmo, M.; Fritz, D. L.; Mergel, J.; Stolten, D. A Comprehensive Review on PEM Water Electrolysis. Int. J. Hydrogen Energy 2013, 38 (12), 4901-4934.

(8) Wiberg, G. K. H.; Fleige, M.; Arenz, M. Gas Diffusion Electrode Setup for Catalyst Testing in Concentrated Phosphoric Acid at Elevated Temperatures. Rev. Sci. Instrum. 2015, 86 (2), 024102.

(9) Inaba, M.; Jensen, A. W.; Sievers, G. W.; Escudero-Escribano, M.; Zana, A.; Arenz, M. Benchmarking High Surface Area Electrocatalysts in a Gas Diffusion Electrode: Measurement of Oxygen Reduction Activities under Realistic Conditions. Energy Environ. Sci. 2018, 11 (4), 988-994.

(10) Alinejad, S.; Inaba, M.; Schröder, J.; Du, J.; Quinson, J.; Zana, A.; Arenz, M. Testing Fuel Cell Catalysts under More Realistic Reaction Conditions: Accelerated Stress Tests in a Gas Diffusion Electrode Setup. J. Phys. Energy 2020, 2 (2), 024003.

(11) Schröder, J.; Quinson, J.; Mathiesen, J. K.; Kirkensgaard, J. J. K.; Alinejad, S.; Mints, V. A.; Jensen, K. M. Ø.; Arenz, M. A New Approach to Probe the Degradation of Fuel Cell Catalysts under Realistic Conditions: Combining Tests in a Gas Diffusion Electrode Setup with Small Angle X-Ray Scattering. J. Electrochem. Soc. 2020, 167 (13), 134515.

(12) Yarlagadda, V.; McKinney, S. E.; Keary, C. L.; Thompson, L.; Zulevi, B.; Kongkanand, A. Preparation of PEMFC Electrodes from Milligram-Amounts of Catalyst Powder. J. Electrochem. Soc. 2017, 164 (7), F845-F849.

(13) Pan, L.; Ott, S.; Dionigi, F.; Strasser, P. Current Challenges Related to the Deployment of Shape-Controlled Pt Alloy Oxygen Reduction Reaction Nanocatalysts into Low Pt-Loaded Cathode Layers of Proton Exchange Membrane Fuel Cells. Curr. Opin. Electrochem. 2019, 18, 61-71.

(14) Bernt, M.; Hartig-Weiß, A.; Tovini, M. F.; El-Sayed, H. A.; Schramm, C.; Schröter, J.; Gebauer, C.; Gasteiger, H. A. Current Challenges in Catalyst Development for PEM Water Electrolyzers. Chem. Ing. Tech. 2020, 92 (1-2), 31-39.
(15) Oakton, E.; Lebedev, D.; Povia, M.; Abbott, D. F.; Fabbri, E.; Fedorov, A.; Nachtegaal, M.; Copéret, C.; Schmidt, T. J. IrO2-TiO2: A High-Surface-Area, Active, and Stable Electrocatalyst for the Oxygen Evolution Reaction. ACS Catal. 2017, 7 (4), 2346-2352.

(16) Reier, T.; Oezaslan, M.; Strasser, P. Electrocatalytic Oxygen Evolution Reaction (OER) on $\mathrm{Ru}, \mathrm{Ir}$, and Pt Catalysts: A Comparative Study of Nanoparticles and Bulk Materials. ACS Catal. 2012, 2 (8), 1765-1772.

(17) Oh, H. S.; Nong, H. N.; Reier, T.; Bergmann, A.; Gliech, M.; Ferreira De Araújo, J.; Willinger, E.; Schlögl, R.; Teschner, D.; Strasser, P. Electrochemical Catalyst-Support Effects and Their Stabilizing Role for IrOx Nanoparticle Catalysts during the Oxygen Evolution Reaction. J. Am. Chem. Soc. 2016, 138 (38), 12552-12563.

(18) Alia, S. M.; Rasimick, B.; Ngo, C.; Neyerlin, K. C.; Kocha, S. S.; Pylypenko, S.; Xu, H.; Pivovar, B. S. Activity and Durability of Iridium Nanoparticles in the Oxygen Evolution Reaction. J. Electrochem. Soc. 2016, 163 (11), F3105-F3112.

(19) Alia, S. M.; Shulda, S.; Ngo, C.; Pylypenko, S.; Pivovar, B. S. Iridium-Based Nanowires as Highly Active, Oxygen Evolution Reaction Electrocatalysts. ACS Catal. 2018, 8 (3), 2111-2120.

(20) El-Sayed, H. A.; Weiß, A.; Olbrich, L. F.; Putro, G. P.; Gasteiger, H. A. OER Catalyst Stability Investigation Using RDE Technique: A Stability Measure or an Artifact? J. Electrochem. Soc. 2019, 166 (8), F458-F464.

(21) Hartig-Weiss, A.; Tovini, M. F.; Gasteiger, H. A.; El-Sayed, H. A. OER Catalyst Durability Tests Using the Rotating Disk Electrode Technique: The Reason Why This Leads to Erroneous Conclusions. ACS Appl. Energy Mater. 2020, 3, 10323.

(22) Lu, Y.; Wang, W.; Xie, F. Investigation of Oxygen Evolution Reaction Kinetic Process and Kinetic Parameters on Iridium Electrode by Electrochemistry Impedance Spectroscopy Analysis. J. Electroanal. Chem. 2020, 871, 114281.

(23) Spöri, C.; Kwan, J. T. H.; Bonakdarpour, A.; Wilkinson, D. P.; Strasser, P. The Stability Challenges of Oxygen Evolving Catalysts: Towards a Common Fundamental Understanding and Mitigation of Catalyst Degradation. Angewandte Chemie - International ed. 2017, 56, 5994-6021.

(24) Rakousky, C.; Reimer, U.; Wippermann, K.; Carmo, M.; Lueke, W.; Stolten, D. An Analysis of Degradation Phenomena in Polymer Electrolyte Membrane Water Electrolysis. J. Power Sources 2016, 326, $120-128$

(25) Bühler, M.; Holzapfel, P.; McLaughlin, D.; Thiele, S. From Catalyst Coated Membranes to Porous Transport Electrode Based Configurations in PEM Water Electrolyzers. J. Electrochem. Soc. 2019, 166 (14), F1070-F1078.

(26) Zhang, G.; Wang, H.; Yang, J.; Zhao, Q.; Yang, L.; Tang, H.; Liu, C.; Chen, H.; Lin, Y.; Pan, F. Temperature Effect on Co-Based Catalysts in Oxygen Evolution Reaction. Inorg. Chem. 2018, 57 (5), $2766-2772$.

(27) Parthasarathy, A.; Srinivasan, S.; Appleby, A. J.; Martin, C. R. Temperature Dependence of the Electrode Kinetics of Oxygen Reduction at the Platinum/Nafion ${ }^{\circledR}$ Interface-A Microelectrode Investigation. J. Electrochem. Soc. 1992, 139 (9), 2530-2537.

(28) Bratsch, S. G. Standard Electrode Potentials and Temperature Coefficients in Water at 298.15 K. J. Phys. Chem. Ref. Data 1989, 18 (1), $1-21$.

(29) Suermann, M.; Schmidt, T. J.; Büchi, F. N. Comparing the Kinetic Activation Energy of the Oxygen Evolution and Reduction Reactions. Electrochim. Acta 2018, 281, 466-471. 